\title{
Philosophiques
}

\section{Morale objective et loi naturelle}

\section{Léonard Ducharme}

Volume 4, numéro 1, avril 1977

URI : https://id.erudit.org/iderudit/203065ar

DOI : https://doi.org/10.7202/203065ar

Aller au sommaire du numéro

Éditeur(s)

Société de philosophie du Québec

ISSN

0316-2923 (imprimé)

1492-1391 (numérique)

Découvrir la revue

Citer ce document

Ducharme, L. (1977). 1. Morale objective et loi naturelle. Philosophiques, 4(1),

102-109. https://doi.org/10.7202/203065ar

Ce document est protégé par la loi sur le droit d'auteur. L'utilisation des services d'Érudit (y compris la reproduction) est assujettie à sa politique d'utilisation que vous pouvez consulter en ligne.

https://apropos.erudit.org/fr/usagers/politique-dutilisation/ 


\title{
1. MORALE OBJECTIVE ET LOI NATURELLE
}

\author{
par Léonard Ducharme
}

En acceptant l'invitation de Louis Valcke de remplacer à cette table le professeur Germain Dandenault, je me suis placé dans une position de liberté absolue. Je n'ai jamais enseigné la morale et je ne prévois pas devoir l'enseigner jamais. Je me trouve donc dans la situation, enviable entre toutes, de pouvoir dire aux autres comment s'acquitter d'une tâche à laquelle je ne devrai jamais faire face moi-même. Cette liberté m'enivre et j'entends bien en abuser de façon éhontée.

Le sujet proposé à notre discussion, l'enseignement de la morale et la question de ses fondements, porte un double sens : philosophique, il provoque à la réflexion, pédagogique, il invite à inventer une stratégie dans la présentation des fruits de cette réflexion. Bien des professeurs, et pas seulement de philosophie, sont allés droit au désastre en se contentant d'avoir raison, sans se préoccuper d'obtenir au moins l'attention sympathique de leurs étudiants. Il va de soi que stratégie ne signifie ni stratagème, ni duplicité, ni, encore moins, malhonnêteté. Mais l'héroïsme solitaire et grandiose du professeur préparant un programme d'enseignement, dans la certitude de n'être même pas entendu, n'exerce sur moi aucun attrait et, même si mon avenir professionnel n'est pas en cause, je n'ose pas proposer aux autres cet austère projet. Je vais donc tenter de soumettre à votre discussion une façon possible d'enseigner la morale, digne, me semble-t-il, d'être dite philosophique et susceptible de recevoir un accueil sympathique, même si elle doit provoquer d'ardentes controverses, ce qui m'a toujours semblé être la marque d'un enseignement de qualité.

On m'a suggéré d'explorer la possibilité de fonder l'enseignement d'une morale objective sur la loi naturelle. Je me suis rallié à cette idée. Ce faisant, je ne peux réclamer d'autre titre à l'originalité que celui dont se parent depuis un certain temps quelques-uns de nos prophètes contemporains, ramenés au millénarisme par l'imminence de l'an deux mille. J'espère avoir plus de titre au respect que je ne leur en trouve moi-même. J'aborderai d'abord le problème pédagogique de la présentation de cette morale, pour toucher ensuite le problème philosophique, celui de sa conception. 
L'idée de loi naturelle a connu bien des protagonistes. J'ai relu les textes de quelques-uns d'entre eux. Ils s'entendent tous pour reconnaître à cette loi naturelle trois attributs, trois propriétés pour reprendre leur vocabulaire. La loi naturelle est universelle, elle est immuable et la connaissance de ses préceptes premiers vient spontanément à une intelligence humaine non déformée, même si la découverte de ses préceptes se fait progressivement, lentement et même si leur expression peut varier d'un groupe humain à l'autre, d'un âge à l'autre. En partant de ces propriétés, il me semble possible de proposer de nos jours un enseignement moral fondé sur la loi naturelle. Il suffit d'amener les étudiants à voir que, de fait, ils vivent de cette vision d'une série de préceptes, s'imposant à tous les esprits de bonne foi, immuables et revêtant à notre époque un caractère d'urgence toute spéciale.

Ce phénomène s'impose avec une évidence spéciale quand on s'arrête aux normes de moralité publique auxquelles nous reconnaissons tous les propriétés caractéristiques de la loi naturelle. Pour l'illustrer, je commenterai brièvement la "Déclaration des droits de l'homme et du citoyen ", votée en août 1789 par l'Assemblée nationale de la Révolution française naissante, et quelques autres textes apparentés. "Préambule: Les représentants du Peuple français, constitués en Assemblée nationale, considérant que l'ignorance, l'oubli ou le mépris des droits de l'homme sont les seules causes des malheurs publics et de la corruption des gouvernements, ont résolu d'exposer dans une Déclaration solennelle les droits naturels, inaliénables et sacrés de l'homme [...] En conséquence, l'Assemblée nationale reconnaît et déclare solennellement, en présence et sous les auspices de l'Être suprême, les droits suivants de l'homme et du citoyen : Article premier : Les hommes naissent et demeurent libres et égaux en droit ; les distinctions sociales ne peuvent être fondées que sur l'utilité commune. " Les droits qu'on reconnaît et déclare appartiennent à tout homme dès sa naissance. Nous connaissons la parenté étymologique entre naissance et $n a$ turel. Il allait de soi que ces droits soient dits naturels, inaliénables, la présence et l'auspice de l'Être suprême les faisaient voir comme sacrés. Faisant abstraction de cette présence et de ces auspices, on les peut encore voir comme naturels et inaliénables. Ces droits, on a bien pu les ignorer, les oublier, les violer, ils étaient toujours là, 
inaliénables. La seule tâche qui s'impose à l'esprit est de les reconnaître après un long oubli et de les déclarer. Déjà en 1774 , les Pères de la Révolution américaine, tout imbus des idées de John Locke, avaient parlé de "vérités évidentes de soi " (self-evident trutbs). L'Assemblée générale des Nations Unies (10 décembre 1948) est revenue à l'idée de "reconnaissance de la dignité inhérente à tous les membres de la famille humaine et de leurs droits égaux ». L'Assemblée nationale du Québec, trouvant peut-être cet "inhérente " trop peu affirmatif, adopte résolument un vocabulaire à relents plus philosophiques et dit "intrinsèque ": "Tout être humain possède des droits et libertés intrinsèques, destinés à assurer sa protection et son épanouissement".

Ces textes et bien d'autres de même venue sont évidemment politiques. Ils évoquent tous le spectre de la tyrannie et des malheurs publics qu'elle provoque: oppression de l'homme par l'homme, révolutions, etc. Il est facile d'être cynique devant toutes ces "déclarations", quand on lit l'histoire des différents régimes qui en ont été ou en sont encore les auteurs. Il me semble difficilement contestable que cette phraséologie, qu'on peut avoir le droit de trouver creuse, exprime la lente prise de conscience par l'humanité de la valeur et des droits de chaque être humain, résultant du simple fait qu'il est humain. Les partisans de la loi naturelle nous avaient prévenus que les préceptes de cette loi se révèlent progressivement à l'esprit. Le mouvement de libération de l'homme, provoqué par tous les excès de bien des régimes dictatoriaux et culminant dans les monarchies absolues du XVIIIe siècle, a amené l'éclatement des théories de l'origine divine du pouvoir politique. John Locke a bien pu donner le coup de grâce philosophique à ces théories, en réfutant un de leurs derniers interprètes, Fullmer, le sauvant ainsi de l'oubli où il aurait sans doute sombré, la conscience populaire grondait déjà de protestations dont il fallait prévoir qu'elles seraient irrésistibles. Les philosophes de l'âge des Lumières n'ont fait que prêter aux cris de cette conscience une expression plus rigoureuse. Depuis lors, les préceptes fondamentaux de cette loi naturelle ont éclaté au grand jour, même quand le clair-obscur a remplacé la lumière dans les écrits philosophiques, à quoi je reviendrai. Nos timidités épistémologiques n'entraveront pas la marche en avant des convictions populaires à trouver un sens d'évidence absolue et immédiate à toutes ces déclarations des droits 
naturels de l'homme. Les yeux de la conscience humaine se sont dessillés une fois pour toutes.

Et même les yeux de notre propre conscience professionnelle. Le monde des écoles grouille de défenseurs des droits naturels. La guerre du Vietnam nous a galvanisés en d'interminables manifestes et manifestations, et seule la fatigue engendrée par cette longue lutte a réduit à des murmures nos protestations contre l'ingérence de la Russie et de Cuba dans les affaires internes du pays angolais. Il nous fallait évidemment surveiller nos voisins du Sud et nous avons laissé à John Diefenbaker le soin de donner une voix à la défense du droit de chaque peuple à disposer librement de luimême. Mais l'énergie nous revient dès que nous sentons menacées toutes nos inaliénables libertés, surtout la liberté qui est notre apanage professionnel, "celle qu'académique on nomme". Et il nous semblerait sans doute indigne de la raison qui parle par nous de réclamer le respect de cette liberté uniquement au nom du droit du plus fort ou du plus tapageur.

Il devrait donc être relativement facile de fonder un enseignement de la morale publique sur la loi naturelle. Nos étudiants ont grandi dans un climat de réclamation du respect des droits égaux pour tous, innés, inaliénables, naturels. Droits et devoirs étant solidaires, la reconnaissance de ces droits entraîne l'obligation de les respecter chez autrui et la dialectique des droits et des devoirs, du bien et du mal, dont vit toute morale, est engagée de fait. Engagée sous le signe de l'inviolabilité, du caractère intrinsèque, naturel des deux termes de cette dialectique.

La conception et l'expression d'une morale personnelle de même inspiration pourront apparaître comme plus difficiles. Le roi de Saint-Exupéry en avait prévenu le Petit Prince. Sa Mejesté venait de créer l'amant d'une rose capricieuse ministre de la justice. Le Petit Prince lui fit remarquer, respectueusement, car c'était un monarque absolu, qu'il n'y avait personne à juger sur la royale planète. "Tu te jugeras toi-même, lui répondit le roi. C'est le plus difficile. Il est bien plus difficile de se juger soi-même que de juger autrui." De fait, chacun se juge soi-même, en fonction d'une image plus ou moins idéale de ce qu'il veut être. Que cette image soit la vision de l'homme dont s'inspire notre morale publique et nos jugements seront fondés sur la loi naturelle. 
Nous pouvons tous, dans notre comportement, avilir cette image que nous demandons aux autres de respecter. Le Roi de Saint-Exupéry rejoint John Locke et bien d'autres quand il admet que l'autorité ne peut être légitime à moins de commander au nom de la raison. En effet, les partisans de la loi naturelle confient tous à la raison de découvrir et d'édicter les préceptes de la loi naturelle, auxquels elle devra ensuite se soumettre elle-même, se trouvant ainsi à la fois législatrice et sujette. Cette raison devra évidemment respecter les raisons du coeur dont on nous assure qu'elle ne les comprend point. La tâche me semble moins impossible que Pascal ne le prétend. Il est donc possible de fonder un enseignement de la morale personnelle sur la loi naturelle. Au sein du fouillis de pulsions instinctives, souvent contradictoires, amour et haine, colère et tendresse, cupidité et générosité, volonté de puissance et attendrissement devant les faibles, la raison devra, laborieusement peut-être, dégager ce coeur de notre être, de notre nature, devant lequel nous exigeons que la puissance politique s'incline et exiger ensuite de chacun qu'il lui manifeste un égal respect, une égale soumission. Cette soumission pourra exiger des réajustements cent fois repris d'habitudes tenaces, des résistances coûteuses aux poussées les plus chargées de promesses ou aux édits des procédés de libération les plus à la mode. Elle pourra amener des lendemains de regrets confus à certaines journées enivrantes. La morale personnelle fondée sur la loi naturelle s'opposerait avec autant de vigueur à l'avilissement de l'homme par lui-même qu'elle le fait à la domination de l'homme par l'homme. Le pharisaïsme menacerait celui qui voudrait qu'on respecte en lui la figure de l'homme, de la nature humaine, s'il la ravageait de ses propres mains.

Tout cela revient à dire qu'une morale fondée sur la loi naturelle ne peut se formuler à moins de s'exprimer en fonction d'une anthropologie. Experte en art pédagogique, elle doit être le fruit d'une réflexion philosophique.

\section{II}

Je serai bref sur ce point. Philosophes, nous avons longtemps parlé de la nautre humaine avec une calme assurance. Tout nous était clair de l'homme, de sa nature, de ses propriétés, de ses droits et devoirs. Les poètes ont souvent été plus modestes que nous. 
Témoin, la Ballade des Menus, propos de Villon, l'un des nôtres en escolâtrie :

Je connais bien mouches en lait,

Je connais à la robe l'homme,

Je connais le beau temps du laid,

Je connais au pommier la pomme,

Je connais l'arbre à voir la gomme,

Je connais quand tout est de mesmes,

Je connais qui besogne et chomme,

Je connais tout, fors que moi-mesme

Je connais pourpoint au collet,

Je connais le moine à la gonne,

Je connais le maître au valet,

Je connais au voile la nonne,

Je connais quand pipeur jargonne,

Je connais fous nourris de crême,

Je connais le vin à la tonne,

Je connais tout, fors que moi-mesme.

Je connais cheval et mulet,

Je connais leur charge et leur somme,

Je connais Biatrix et Belet,

Je connais jet qui nombre et somme,

Je connais vision et somme,

Je connais la faute des Boemes,

Je connais le pouvoir de Rome,

Je connais tout, fors que moi-mesme.

Prince, je connais tout en somme,

Je connais coulourés et blêmes,

Je connais mort qui tout consomme,

Je connais tout, fors que moi-mesme.

Ceux de mes collègues dont je trace le programme d'action voudront sans doute éviter l'optimisme confiant de bien de nos prédécesseurs parlant de la nature humaine avec l'assurance née de la familiarité la plus intime ; toutes les natures, l'humaine avec les autres, leur étaient si facilement accessibles. Donnée d'un seul coup à leur raison, elle fondait sur son immédiate évidence toutes les déductions dont ils avaient le désir. Nous sommes devenus plus circonspects, à la suite de maintes déconfitures. Personnellement, je 
ne trouve rien dans la nature humaine qui puisse justifier l'usage de termes comme "famille humaine ", "esprit de fraternité " employés par la déclaration de l'ONU, si on veut parler philosophiquement et dépasser la métaphore. Je ne me sens pas le droit de réclamer une attitude fraternelle de qui que ce soit, à moins qu'il ne soit né du même père que moi. La chaleur universelle de nos milieux universitaires me suggère bien parfois l'audace de penser à une famille, au moins à une confrérie. Hélas! même dans ces moments d'euphorie, ma conscience épistémologique crie : métaphore!

Mais nous avons exagéré nos prudences. Une immense modestie collective s'est emparée de nous. La raison, humiliée, s'est mise à s'avilir elle-même et nous avons prêté allégrement toutes nos complicités épistémologiques à cette oeuvre d'autodestruction. Les mots nature et naturel nous sont devenus indécents. Mikel Dufrenne écrivait en 1968, au début de la seconde partie de son livre Pour l'bomme: "Nous avons parcouru les thèmes principaux qui orientent la pensée contemporaine vers le reniement de l'homme. [ . . . . Cette entreprise, pourtant, n'est-elle pas dérisoirement présomptueuse? La vie et l'histoire n'ont jamais attendu la philosophie, et l'homme se défend très bien tout seul. [ . . . . Au vrai, il s'agit moins ici de défendre l'idée de l'homme que le droit de la philosophie. Car le destin de la philosophie est lié à l'intérêt qu'elle porte à l'homme. " $\grave{A}$ la fin du même ouvrage, parlant des créations de la technique contemporaine, Dufrenne ajoute : " La nature naturée par l'homme, témoigne encore de la Nature naturante. "

Retenons l'avertissement : "La vie et l'histoire n'ont jamais attendu la philosophie. "Notre époque vit de la conviction que l'homme est d'une nature particulière à laquelle sont attachés des droits et une dignité inaliénables. Bien des pudeurs nous sont venues qui nous font hésiter à parler philosophiquement de cette nature. La vie n'attendra pas et nous laissera pour compte, bien en arrière d'elle. D'autant plus que nous réclamons pour nous-mêmes des droits inaliénables. Consommer durant nos cours le divorce entre notre pensée professionnelle et nos renvendications professionnelles nous accule à des inconséquences devant lesquelles, philosophes, nous devrions pâlir. 
Sans revenir à nos antiques présomptions, nous pouvons certainement reconnaître que nos complaisances actuelles à collaborer à l'oeuvre de la destruction de l'homme, et de la philosophie, ont assez duré. Nous pouvons nous remettre à la tâche de dire ce qu'est l'homme et sa nature, dans une anthropologie ouvertement philosophique, rendant ainsi possible l'expression d'une morale fondée sur la loi naturelle. Il nous faudra évidemment accepter l'aide de ce qu'on appelle les sciences humaines. Ces sciences ont même mère que la philosophie, l'intelligence, la raison, elles peuvent donc nous tendre une main fraternelle. Et que l'on ne me dise pas que je chavire dans la métaphore en parlant de l'intelligence, de la raison comme d'une mère. Ce n'est pas moi qui le premier l'ai vue en train de concevoir et je ne m'agenouille plus devant le berceau d'où ses concepts tyranniques prétendent régir la pensée.

Cette anthropologie philosophique pourrait bien, de temps en temps, ressentir le besoin d'un point d'appui métaphysique. Il subsiste parmi nous des entêtés nostalgiques, tout disposés à tendre une main fraternelle à qui veut la prendre, même si on accuse parfois la métaphysique de bâtardise.

\section{UNIVERSITÉ D'OTTAWA}

